\title{
Uso de fármacos como alternativa de intervención en niños con problemas de déficit de atención
}

DOI: https://doi.org/10.32870/dse.v0i8.314

Reynalda Armida Beltrán Quintero*

M es un chico de 15 años cuyos maestros coinciden en que siempre está sentado al fondo del salón, es indolente, su actitud es poco cooperadora, raras veces toma nota, solo entrega algunas tareas y se irrita cuando el maestro le pide que conteste alguna pregunta o le llama la atención. Por el contrario, suele observar una actitud respetuosa hacia sus mayores y obtiene buenas notas en los exámenes. Al concluir la evaluación, se diagnosticó тDAн (Trastorno por déficit de atención con hiperactividad).

¿Podrá ser eso posible en un joven que a primera vista simplemente parece estar poco motivado o cuyos padres no supervisan?

La descripción siguiente nos resulta más familiar:

Al momento de entrar al salón de clases, A, llega gritando, jugando o molestando a algún compañero, se le dificulta seguir las reglas del salón, permanecer sentado, respetar su material y el de los demás, sus períodos de atención son muy cortos en la mayoría de actividades; en las clases de inglés y deportes es muy difícil que ponga atención y que se mantenga sentado en el tapete, además tiene actitudes agresivas hacia sus compañeros.

La anterior es la típica manera en que consideramos al niño con тDAн lo cual no es muy grave, la siguiente es otra descripción del cuadro típico de un niño con TDAH, hecha por la madre:

Es un niño con una inteligencia de genio pero es muy inquieto, no para de molestar para llamar la atención, es muy negativo, tiene muy malas maneras en la mesa, se enoja, se pone rojo, no sabe lo que hace ni lo que yahoo.com

* Neuróloga pediatra, Unidad Integral de Neurociencias Guadalajara, Jalisco. Correo electrónico: reynarmida@ 
dice y llega a decir cosas muy feas y a pegar, es mañoso, es muy quisquilloso. En el colegio no trabaja, es muy negativo, es muy contestón, es flojo, da lo mínimo, todo discute. Le falta al respeto a la maestra, se sale del salón, para él todo es "chafa". Le encanta aparecer como "bobo", le encanta hacer historias.

\section{Entonces, ¿qué es el TDAH?}

El тDAн es una alteración de los circuitos neurales que regulan la atención y el autocontrol, y modulan la emoción. Es una condición biológica, altamente heredable que se manifiesta por tres síntomas medulares; inatención, hiperactividad e impulsividad, cuya expresión clínica se ve influida por factores asociados inherentes al individuo tales como coeficiente intelectual, problemas de aprendizaje, enfermedades asociadas, etcétera, así como a factores ambientales, sociales y familiares (APA, 2013). Desde la perspectiva de funcionamiento neuropsicológico es una condición altamente compleja y de origen multifactorial. No se trata tan sólo de falta de interés sino de una incapacidad para orientar y mantener la atención cuando la tarea es impuesta o se vuelve repetitiva, difícil o poco gratificante. Esto se entiende si consideramos que la atención es un proceso cognitivo complejo y dinámico, que requiere la concertación de numerosas funciones de la corteza cerebral que interactúan en infinitas combinaciones segundo a segundo, a fin de editar el comportamiento adecuado a las circunstancias actuales. "Prestar atención" no se limita a observar fijamente un evento en particular sino un acto continuo y cambiante de enfocar, jerarquizar, enfocar selectivamente, regular el nivel de alerta, mantener el esfuerzo y adecuar la velocidad de procesamiento del cerebro a las demandes del entorno. Además involucra el control y modulación de las emociones, el uso de la memoria de corto y largo plazo y el mantener la tarea en perspectiva (Brown, 2002).

El considerar al niño con déficit de atención bajo este paradigma explica las manifestaciones tan diversas de los casos anteriores:

\section{Tendencia a postergar:}

Tienen dificultad en organizar tareas y materiales, en estimar el tiempo, establecer prioridades e iniciar la actividad; pasan demasiado tiempo sacando punta o buscando sus materiales, no concluyen a tiempo o no entregan tareas.

2. Se distraen con estímulos irrelevantes y pierden fácilmente el rumbo:

No concluyen el trabajo de clase o no afinan detalles (título, fecha, uso de dos tintas, etcétera).

3. Se aburren fácilmente o experimentan sueño: "no les gusta leer", juegan durante la clase, garabatean, lucen desinteresados.

4. Dificultad crónica para el manejo de la frustración, la ira, la ansiedad, la desilusión, el deseo y otras emociones.

Se irritan fácilmente, pierden el interés ante la mínima dificultad, lucen poco orientados hacia el logro, tienen cambios bruscos de humor. 


\section{Dificultad con la memoria a corto plazo.}

No siguen las rutinas diarias a menos que se les recuerde continuamente. Olvidan fácilmente lo aprendido si lo dejan de ejercitar (los algoritmos de las operaciones aritméticas, por ejemplo). Memorizan con dificultad las tablas de multiplicar.

6. Impulsividad. Dificultad en regular el ritmo de sus acciones.

Hacen y dicen cosas sin pensar y se meten en problemas por eso: interrumpen la clase, son los bufones del salón, hacen comentarios inapropiados, interfieren con el trabajo de los otros, no esperan a escuchar o leer todas las instrucciones antes de comenzar, etcétera.

Existen suficientes evidencias científicas (Sharma, 2014) de la incapacidad para lograr esta tarea compleja que obedece a una disponibilidad ineficiente (y no ausencia) de los neurotransmisores necesarios para conectar la red neural correspondiente para adecuarnos a las circunstancias. Esto explica por qué cuando los estímulos son reducidos y/o fáciles, altamente gratificantes, presentados en la frecuencia adecuada; o las señales externas son precisas; o existe un control externo que ayude a regular la emoción y/o redirigir la acción; el individuo presenta un comportamiento apropiado a las circunstancias; sin embargo, esto no es lo que suele suceder en la vida cotidiana.

Como es de suponer es difícil que esto suceda regularmente en el aula y durante la convivencia. Mientras el alumno atiende la clase deberá procesar cada una de las palabras dichas por su profesor y ponerlas en el contexto actual de la comunicación. Para hacerlo, deberá utilizar, además de los procesos lingüísticos, recursos de procesamiento viso-espacial, temporosecuencial y memoria, así como regular la acción a fin de interrumpir oportuna y adecuadamente para solicitar una aclaración, pedir una tregua, etcétera. Y no se diga cuando tiene que trabajar independientemente, en estas circunstancias deberá tener en la memoria inmediata la información relevante para la tarea en curso, tener sentido del tiempo, recordar las reglas y mantener un esfuerzo constante.

En el patio de recreo la situación es aún más compleja. La conducta impulsiva en situaciones sociales, se manifiesta por comentarios imprudentes o reacciones poco amables. Estas conductas molestan a los demás, no son productivas en situaciones de juego y provocan poca aceptación o inclusive rechazo. A su vez, el niño con тDAн malinterpreta o no toma en cuenta las pautas implícitas en la situación, otras veces no las reconocen o inclusive distorsionan el significado del lenguaje no verbal de quienes los rodean. Son incapaces de atender las pistas no verbales de la expresión facial, el lenguaje corporal y las inflexiones del lenguaje, además tienen escasa capacidad para ponerse en el lugar del otro o de compartir su estado de ánimo. Debido a su hiperactividad e impulsividad, se entrometen en las conversaciones, cambian abruptamente de tópico o ignoran la regla habitual de tomar turno en una conversación. La torpeza en el discurso origina que sus pares los rechacen e inclusive el que sean ridiculizados o engañados (Beltrán, 2013). 
El TDAH tiene un gran impacto sobre la calidad de vida del paciente, lo cual es evidente en un rendimiento académico pobre. Usualmente estos niños requieren clases particulares o ser puestos en clases especiales, cambios frecuentes de escuela por mala conducta y eventualmente deserción escolar. Estos chicos enfrentan serias dificultades en las relaciones interpersonales y aumento del riesgo para conductas adictivas y accidentes, debido a su impulsividad. Para la familia implica aumento del estrés intrafamiliar, mayor predisposición a la depresión en los padres, aumento de problemas maritales e impacto en el rendimiento profesional de los mismos, además, es definitivo un incremento significativo en la carga económica (Cantwell, 1996).

El tratamiento del тDAн debe ser multimodal y los objetivos son mejorar el funcionamiento del niño en casa, en la escuela y con sus compañeros, a través de modificar su inatención, impulsividad e hiperactividad. Además, se trata de maximizar las funciones cognitivas, las habilidades socioadaptativas y la autoestima.

La medicación ha sido el pilar del tratamiento durante varias décadas. Los efectos favorables de las anfetaminas se descubrieron por casualidad, por Charles Bradley en 1937 (Bradley, 1937). El metilfenidato (MF) fue aprobado en Estados Unidos en 1955 y las evidencias, acumuladas hasta hoy, dejan pocas dudas de que el tratamiento farmacológico ha tenido un impacto beneficioso sobre las manifestaciones conductuales centrales del TDAH, puesto que reducen los síntomas medulares en forma inmediata 80 por ciento, en el plazo mediato mejoran el rendimiento escolar y reducen la conducta oposicionista desafiante y tiene un perfil costo/beneficio favorable cuando se compara con los beneficios y costos de no utilizar intervenciones farmacológicas (Plizka, et al., 2006). No obstante, aún quedan sin resolver algunas dudas sobre la amplitud de sus efectos terapéuticos, su capacidad para reducir acontecimientos adversos como el trastorno por consumo de sustancias, la conducta antisocial y el fracaso escolar y su papel en el tratamiento de poblaciones especiales como los niños menores de 6 años o los casos con una función intelectual baja.

Las opciones terapéuticas siguen siendo asunto de debate a la luz de los posibles efectos secundarios. Afortunadamente existen guías de tratamiento que ayudan al médico a elegir un tratamiento cuya eficacia y seguridad estén basados en evidencia (AACAP, 2002; International Consensus, 2004; NIH, 2000 y Vázquez et al., 2010).

Los fármacos disponibles en Estados Unidos y Canadá se agrupan en la tabla 1, en la que se destacan en negrita los disponibles en México.

Los medicamentos de primera línea son los estimulantes y la atomoxetina. La elección depende de las características individuales del caso, la disponibilidad y el costo del medicamento y los prejuicios parentales sobre los fármacos. Independientemente de la elección, el resultado debe ser efectivo en controlar los síntomas medulares a corto plazo, de optimizar el funcionamiento escolar y social a mediano plazo y facilitar la adquisición y consolidación de las funciones ejecutivas a largo plazo. 


\section{Conclusión}

El TDAH es un trastorno neuropsiquiátrico de origen multifactorial, que se traduce en una dificultad en orientar y mantener la atención y regular la emoción manifiesta en todos los contextos y a lo largo de la vida. No tratada representa un riesgo elevado de fracaso escolar y psicopatología. El uso de fármacos oportunamente incrementa la posibilidad de una vida exitosa.

\section{Referencias}

AACAP (2002). Official Action, Practice parameter for the use of stimulant medications in the treatment of children, adolescents, and adults. Journal of American Academy of Child and Adolescent Psychiatry, 41(2) supplement 26S-49S.

APA (2013). Diagnostic and Statistical Manual of Mental Disorders. 5th Edition: DSM-5. American Psychiatric Association.

Beltrán Q., R. A. (2013). Trastorno por déficit de atención y trastorno del desarrollo del Lenguaje. México: Academia Mexicana de Pediatría.

Bradley, C. (1937). The behaviour of children receiving Benzedrine. American Journal of Psychology. 94, p. 577.

Brown, T. E. (2002). DSM IV: ADHD and executive function impairments. Advanced studies in Medicine. 2(25), pp. 910-914.

Cantwell, D. P. (1996). Attention deficit disorder: a review of the past 10 years. Journal of American Academy of Child and Adolescent Psychiatry. 35 (8), pp. 978-987

International Consensus statement on attention-deficit/hyperactivity disorder (2004). (ADHD) and disruptive behaviour disorders (DBDs): clinical implications and treatment practice suggestions, European Neuropsychopharmacology 14, pp.11-28

National Institutes of Health Consensus Development Conference Statement (NIH) (2000). Diagnosis and Treatment of Attention.Deficit/Hiperactiviti Disorder (ADHD) Journal of the American Academy of Child and Adolescent Psychiatry. 39(2), pp. 182-193

Pliszka, S. R., D. C. Glahn, M. Semrud-Clikeman, C. Franklin, R. Perez, J. Xiong J. y M. Liotti (2006). Neuroimaging of inhibitory control areas in children with attention deficit hyperactivity disorder who were treatment naive or in long-term treatment. Am J Psychiatry. 163(6), pp.1052-60.

Sharma, A. y J. Couture (2014). A review of the pathophysiology, etiology, and treatment of attention-deficit hyperactivity disorder (ADHD). Ann Pharmacother. 2014, feb.; 48(2), pp. 209-25

Vázquez, M. J., M. E. Cárdenas, M., Feria, C. Benjet, L. Palacios y F. De la Peña (2010). Guía clínica para el trastorno por déficit de atención e hiperactividad. En Shoshana, J. del B., J. Alfaro y M. E. Medina (eds.). Serie Guías Clínicas para la Atención de Trastornos Mentales. México: Instituto Nacional de Psiquiatría Ramón de la Fuente. 


\section{Cuadro 1 \\ Medicamentos utilizados en Tratamiento del TDAH}

\begin{tabular}{|c|c|c|}
\hline Estimulantes & & \\
\hline Acción corta & Dosis & Duración \\
\hline $\begin{array}{l}\text { Dextroanfetamina } \\
\text { Metilfenidato }\end{array}$ & $0.15-0.4 \mathrm{mg} / \mathrm{kg} /$ dosis dos o tres veces al día & 4 a 6 horas \\
\hline $\begin{array}{l}\text { Metilfenidato } \\
\text { Acción prolonaada }\end{array}$ & $0.30-0.7 \mathrm{mg} / \mathrm{kg} / \mathrm{dosis}$ dos o tres veces al día & $3-4$ horas \\
\hline Anfetamina & $\begin{array}{l}0.6-0.7 \mathrm{mg} / \mathrm{kg} / \mathrm{dosis} \text { por las mañanas o dos } \\
\text { veces }\end{array}$ & 4-8 horas \\
\hline $\begin{array}{l}\text { Metilfenidato SR } \\
\text { Metiffenidato OROS } \\
\text { Metilfenidato Sodas }\end{array}$ & $\begin{array}{l}0.6-1.3 \mathrm{mg} / \text { dosis por las mañanas o dos veces } \\
0.3-2.56 \mathrm{mg} / \mathrm{kg} / \mathrm{dia} \\
1-2 \mathrm{mg} / \mathrm{kg} / \mathrm{d} \text { osis por las mañanas }\end{array}$ & $\begin{array}{l}\text { 4-8 horas } \\
8-12 \text { horas } \\
8-10 \text { horas }\end{array}$ \\
\hline Antidepresivos & & \\
\hline $\begin{array}{l}\text { Imipramina } \\
\text { Nortriptilina } \\
\text { Burpropion } \\
\text { Fluoxetina } \\
\text { Paroxetina } \\
\text { Venlafaxina } \\
\text { Sertralina }\end{array}$ & $\begin{array}{l}1 \text { 1-3-mg/kg/dia dos veces al día } \\
0.5-4 \mathrm{mg} / \mathrm{kg} / \text { /dia dos o tres veces al día } \\
2-6 \mathrm{mg} / \mathrm{kg} / \mathrm{dia} \text { dos o tres veces al dia } \\
10-20 \mathrm{mg} \text { dia } \\
20-40 \mathrm{mg} / \mathrm{dia} \\
1-3 \mathrm{mg} / \mathrm{kg} / \text { dia dos o tres veces al día } \\
12.5 \text { a } 100 \mathrm{mg} / \mathrm{dia} \text { (dosis media de } 50 \mathrm{mg} \text { ) }\end{array}$ & \\
\hline$\underline{\text { Otros }}$ & & \\
\hline $\begin{array}{l}\text { Atomoxetina } \\
\text { Clonidina } \\
\text { Guanfacina } \\
\text { Buspirona }\end{array}$ & $\begin{array}{l}0.5-1.8 \mathrm{mg} \times \mathrm{kg} \times \text { día una vez al día } \\
3-5 \mathrm{mcg} / \mathrm{kg} / \mathrm{dia} \text { tres o cuatro veces por día } \\
50 \mathrm{mcgr} / \mathrm{kg} / \mathrm{dia} \text { dos o tres veces al dia } \\
0.5-1 \mathrm{mg} / \mathrm{kg} / \mathrm{dia}\end{array}$ & $\begin{array}{l}24 \text { horas } \\
2-4 \text { horas } \\
4-8 \text { horas }\end{array}$ \\
\hline Estabilizadores del humor & & \\
\hline $\begin{array}{l}\text { Valproato de sodio } \\
\text { Carbonato de litio } \\
\text { Carbamazepina } \\
\text { Topiramato }\end{array}$ & $\begin{array}{l}15 \text { a } 50 \mathrm{mg} / \mathrm{kg} / \text { día } 2 \text { o } 3 \text { veces al día } \\
0.9 \mathrm{mEq} / \mathrm{l} \\
15-35 \mathrm{mg} / \mathrm{kg} / \text { día } 2 \text { o } 3 \text { veces al día } \\
1-7 \mathrm{mg} / \mathrm{kg} / \mathrm{dia} \text { en dos dosis }\end{array}$ & \\
\hline Neurolépticos & & \\
\hline $\begin{array}{l}\text { Haloperidol } \\
\text { Pimozide } \\
\text { Risperidona }\end{array}$ & 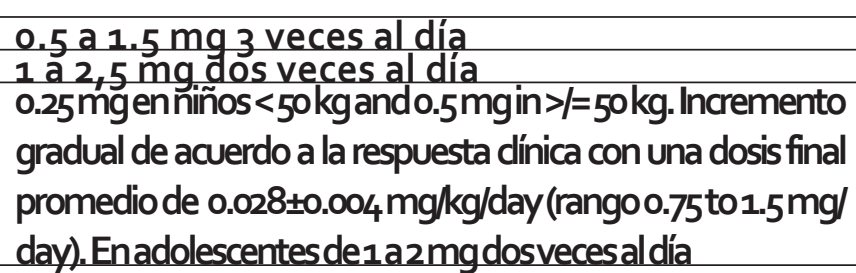 & \\
\hline
\end{tabular}

